\title{
Effects of Different Sleep Restriction Protocols on Sleep Architecture and Daytime Vigilance in Healthy Men
}

\author{
H. WU ${ }^{1 *}$, W. S. STONE ${ }^{2 *}$, X. HSI ${ }^{3,4}$, J. ZHUANG $^{1}$, L. HUANG $^{1}$, Y. YIN $^{1}$, L. ZHANG $^{1}$, \\ Z. ZHAO ${ }^{1}$
}

*Both authors contributed equally to this work

${ }^{1}$ Department of Neurology, Institute of Neuroscience and MOE Key Laboratory of Molecular Neurobiology, Neuroscience Research Center of Changzheng Hospital, Second Military Medical University, Shanghai, P.R. China, ${ }^{2}$ Beth Israel Deaconess Medical Center and Harvard Medical School Departments of Psychiatry, Boston, USA, ${ }^{3}$ Department of Psychiatry, Cambridge Health Alliance / Harvard Medical School, Cambridge, USA, ${ }^{4}$ MIT Medical, Massachusetts Institute of Technology (MIT), Cambridge, USA

Received September 20, 2009

Accepted March 5, 2010

On-line April 20, 2010

\section{Summary}

Sleep is regulated by complex biological systems and environmental influences, neither of which is fully clarified. This study demonstrates differential effects of partial sleep deprivation (SD) on sleep architecture and psychomotor vigilance task (PVT) performance using two different protocols (sequentially) that each restricted daily sleep to 3 hours in healthy adult men. The protocols differed only in the period of sleep restriction; in one, sleep was restricted to a 3-hour block from 12:00 AM to 3:00 AM, and in the other, sleep was restricted to a block from 3:00 AM to 6:00 AM. Subjects in the earlier sleep restriction period showed a significantly lower percentage of rapid-eye-movement (REM) sleep after 4 days (17.0 vs. $25.7 \%$ ) and a longer latency to the onset of REM sleep (L-REM) after 1 day (78.8 vs. $45.5 \mathrm{~min}$ ) than they did in the later sleep restriction period. Reaction times on PVT performance were also better (i.e. shorter) in the earlier SR period on day 4 (249.8 vs. $272 \mathrm{~ms}$ ). These data support the view that earlier-night sleep may be more beneficial for daytime vigilance than later-night sleep. The study also showed that cumulative declines in daytime vigilance resulted from loss of total sleep time, rather than from specific stages, and underscored the reversibility of SR effects with greater amounts of sleep.

\section{Key words}

Sleep restriction • Polysomnogram • Psychomotor vigilance task

\begin{abstract}
Corresponding author
Zhongxin Zhao, Department of Neurology, Institute of Neuroscience and MOE Key Laboratory of Molecular Neurobiology, Neuroscience Research Center of Changzheng Hospital, Second Military Medical University, Shanghai 200433, P.R. China. E-mail: zhongxinzhao@smmu.edu.cn, Fax: +86-218188-5451.
\end{abstract}

\section{Introduction}

As sleep deficits increasingly characterize modern civilization, studies of sleep deprivation (SD) or sleep restriction (SR) are increasingly important (Bonnet and Arand 1995). Sleep disturbances are associated with homeostatic imbalance and circadian disorders, and are related to cognitive, endocrine, immune and cardiovascular dysfunction. Consequently, research on sleep and associated mechanisms has grown consistently, although the roles of sleep remain enigmatic in many respects.

Although polysomnograms (PSG) have been used extensively in sleep deprivation and sleep restriction research, reported changes in sleep architecture under different sleep conditions are inconsistent. For example, Elmenhorst et al. (2008) reported decreased non-REM (NREM) 'lighter' sleep stages (defined as NREM stages 
1 and 2), but increased 'deeper' NREM sleep stages (defined as NREM stages 3 and 4) and rapid eye movement (REM) sleep in healthy males following reductions of sleep (from 8 to 5 hours) for four consecutive nights. However, Webb and Agnew (1974) reported that partial sleep deprivation (PSD) mainly reduced REM sleep and S2 in the last sleep cycle, with minimal effects on $\mathrm{S} 4$.

Multiple factors regulate and/or influence the sleep-wake cycle, and are likely to contribute to variation in experimental results. Sleep is regulated by complex systems (Borbély and Achermann 1999, Greene and Siegel 2004), which include circadian modulation, homeostatic influences, ultradian factors, etc. However, the rules of regulation are not clear. Tilley and Wilkinson (1984) restricted sleep to $4 \mathrm{~h}$ in the first or second half of the night, for two nights, and found that sleep restricted to the second half of the night resulted in higher amounts of REM and stage 4 sleep, and lower amounts of stage 2 sleep, compared to sleep restricted to the first half of the night. Interpretations of these findings, however, are limited by the short 2-day SR period. Recently, another study, which restricted sleep to $4 \mathrm{~h}$ per night for 7 days, showed no significant differences in sleep architecture between earlier-night and later-night SR groups, but revealed a wide range of individual differences in response to the restriction periods (Guilleminault et al. 2003).

We reported previously that early morning hormonal levels (i.e. cortisol) differed according to the timing of the sleep restriction period, with larger reductions in cortisol associated with a 3-hour sleep restriction period earlier in the night (Wu et al. 2008). In the current study, polysomnographic recordings were obtained to determine whether earlier-night and laternight sleep restriction differ in their effects on sleep architecture and on a measure of daytime vigilance (Anderson and Horne 2006). We examined the effects of two 3-hour sleep restriction regimens on sleep architecture, for four consecutive nights (one regimen at a time, separated by a recovery period). Because total sleep time is shortened during the 3-hour SR periods, this report focuses on the relative proportions of each sleep stage within total sleep time (TST) to clarify levels of different sleep stages.

\section{Materials and Methods}

\section{Subject recruitment and selection}

Fourteen healthy, adult Han Chinese males (mean age of $22.1 \pm 1.1$ years) were recruited from a military medical university in China, in part to minimize heterogeneity due to gender or age differences (Philip et al. 2004). Subjects' mean body mass index (BMI) was $22.5 \pm 1.7 \mathrm{~kg} / \mathrm{m}^{2}$. They were all non-smokers and were in general good health physically, with similar living habits and no history of alcohol or other substance dependence. All participants signed informed consent forms before the study. The study was approved by the clinical trial ethics committee of the Hospital.

Before the SR study, subjects were required to sleep in the polysomnography (PSG) laboratory of the medical university. This allowed them to adapt to the environment, and also allowed the researchers to identify and exclude any subjects with sleep disorders. Four such subjects were excluded from the study in this manner, due to sleep-onset insomnia, trouble maintaining sleep, or early morning wakening. The remaining 10 subjects completed the study protocol, with one exception. All PVT data were excluded from one subject who appeared (behaviorally) to experience brief periods of sleep during the PVT on SR 4. This behavior was not observed at other times, and sleep data were not excluded for this subject. As noted, procedures related to hormonal assessments and absolute sleep levels were reported previously (Wu et al. 2008).

\section{Sleep restriction study design}

The timetable and evaluation schedule are shown in Figure 1. Each subject followed two, one-week sleep regimens, separated by a one-week, unrestricted sleep recovery interval. Considering that subjects might be sleep phase-delayed, and that they were accustomed to going to bed between 11:00 p.m. and 12:00 a.m., sleep times of both SR schedules began after midnight (designated as '0:00 a.m.' in this study) to ensure high sleep efficiency (Guilleminault et al. 2003, National Sleep Foundation 2003). The SR protocols consisted of an 'earlier-night' SR (ESR) period (with sleep time from 00:00 a.m. to 03:00 a.m.) and a 'later-night' SR (LSR) period (with sleep time from 03:00 a.m. to 06:00 a.m.). During the SR trials, sleep was not allowed at other times. The duration of each SR regimen was seven nights: the night before SR, four nights of SR and two recovery nights $(\mathrm{RN})$ after SR. Sleep was unrestricted in the baseline and RN periods. Based on the assumption that delaying sleep onset gradually would be easier to tolerate, the earlier-night SR study was conducted first. Between the two SR trials, there was a seven-day 

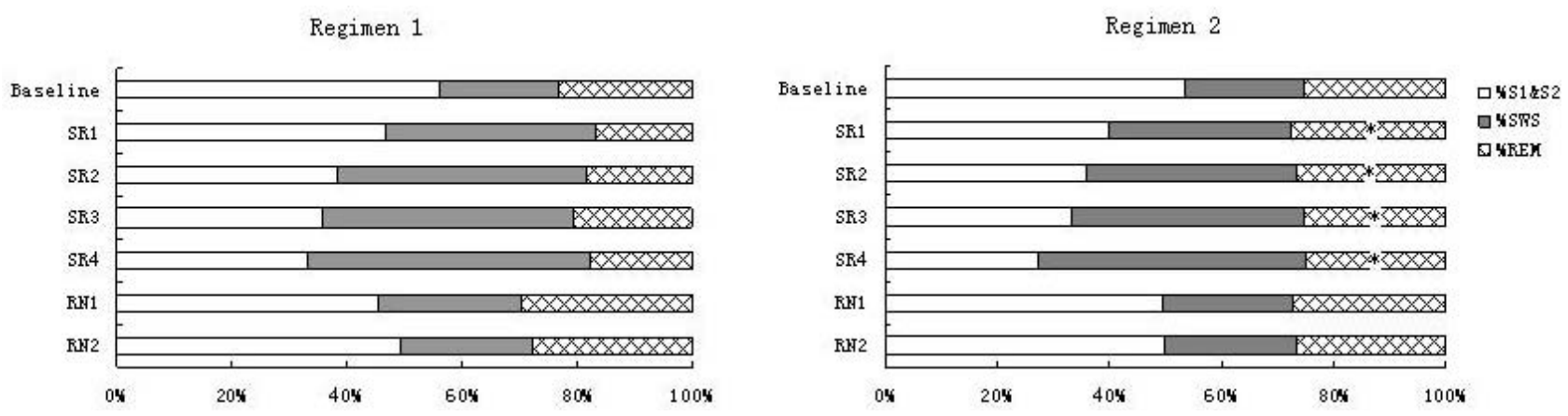

Fig. 1. Changes in the percentage of PSG parameters during the 7-day SR regimens. Regimen 1 - earlier-night sleep restriction period (sleep time 0:00 3:00); Regimen 2 - later-night sleep restriction period (sleep time 3:00 6:00). Unrestricted sleep was recorded in the baseline night before the SR periods and in the two recovery nights after the SR periods. SR - sleep restriction; RN - recovery night; S1\&S2 - Non-REM sleep stages 1 and 2; SWS - slow wave sleep (Non-REM sleep stages 3 and 4); REM - rapid eye movement sleep. Symbol $*$ indicates $\mathrm{p}<0.05$ by paired t-test in comparison with the same elapsed time during later-night $\mathrm{SR}$.

recovery period to ensure a complete return to baseline performance. Overnight polysomnograms (PSG) were continuously recorded during both SR regimens. Performance on the psychomotor vigilance task (PVT) was measured daily at 12:00 p.m.

During the SR trials, subjects stayed in the PSG laboratory at room temperature $\left(22-25{ }^{\circ} \mathrm{C}\right)$. All subjects were instructed to maintain sitting or standing positions during periods of wakefulness. Lying in bed was prohibited except for defined sleep periods. Wakefulness was maintained by exercising physically (walking, bicycling etc), reading, watching television, playing card/computer games, etc. Subjects' activities were under constant supervision by PSG-recorder and by research assistants to ensure wakefulness. During the washout period, subjects continued their normal activities, which were highly regulated because they were students at a military medical university. Nutritionally balanced meals were provided throughout the entire study, and intake of alcohol, nicotine, caffeine, tea or psychoactive drugs was prohibited, to avoid potentially confounding influences on the experimental protocols.

\section{Polysomnographic recording}

During the adaptation nights (weeks 1 and 3), the PSGs included an electroencephalogram (EEG) (C3/A2, C4/A1, O1-O2), an electrooculogram (EOG), a chin and leg electromyogram (EMG), and an electrocardiogram (ECG). Respiration was monitored by measuring nasal flow (nasal cannula/pressure transducer) and breathing through the mouth (thermistor), by thoracic and abdominal bands, and by pulse oximetry. During the experimental nights, only EEG, EOG, chin and leg EMG, and ECG were recorded. Bio-calibrations were always performed prior to each SR trial to ensure that electrodes were positioned properly to record data.

Polysomnographic recordings were analyzed by an experienced researcher following standard scoring criteria (Rechtschaffen and Kales 1968). Sleep onset was defined as the presence of PSG indicators of one or more sleep stages that persisted for $90 \mathrm{~s}$ or longer. The following parameters were obtained for each subject: TST (consisting of all NREM+REM sleep), percentage of total NREM sleep, including all NREM sleep stages (S1\%, S2\%, S3\% and S4\%, which reflects increasing 'deeper' sleep stages), percentage of REM sleep (REM\%), REM sleep latency (L-REM, defined as the time from sleep onset to the beginning of REM sleep), and sleep efficiency (Sl-eff, defined as the amount of sleep divided by the amount of bed time). The term 'slow wave sleep' (SWS) will be used to refer to a combination of the deeper NREM sleep stages 3 and 4 . The acronym 'SOREMP' refers to sleep onset REM periods.

\section{Psychomotor vigilance task (PVT)}

The PVT is a straightforward psychological test designed to assess sustained vigilance by measuring the latency to respond to visual signals (Dinges and Powell 1985). Subjects sat in front of the PVT apparatus, about $30-40 \mathrm{~cm}$ away. Over the course of three 10-min training sessions, they learned to respond rapidly (by hitting a button) to a digital counter that counted in millisecond (ms) increments. Hitting the button stopped and then reset the counter. The duration of the test is $10 \mathrm{~min}$. Reaction time (RT in ms) and lapses (defined as a reaction time $>500 \mathrm{~ms}$ ) were recorded. The PVT program generates mean RTs and lapses, and standard deviations (SD). Subject performance on the PVT was measured daily at 12:00 P.M. We chose this time because it typically comes during a break in the subjects' normal 
routine, in two ways. First, it is a time when the subjects in this study normally receive a break from their daily work. Second, it is a time when many Chinese take naps. It is thus a likely biological nadir for these subjects (Dijk et al. 1992), and a time when they may be relatively vulnerable to conditions that impair vigilance.

\section{Data analysis and statistics}

Baseline levels of sleep (S1\%, S2\%, SWS\%, S4\%, REM\%, L-REM, Sl-eff, TST, SWS) and PVT parameters (PVT-RT and lapses) prior to the two SR trials were compared by paired t-tests. Homogeneity of variance tests were used in all analyses. Dunnett t-tests were used to compare values from corresponding time points (SR1 4 and RN1 2) with baseline values in each SR trial. Pearson correlation analysis was used to analyze relationships between these parameters and the number of days of SR for both conditions. Since data were obtained from (two periodically) repeated measurements of the same subjects, generalized estimating equations (GEE), were used to analyze correlations between the two SR protocols (Hanley et al. 2003). GEEs use a generalized linear model to estimate relatively unbiased regression parameters compared to least squares regression, and are particularly appropriate for these data. This method of analysis was useful in our previous study ( $\mathrm{Wu}$ et al. 2008). Paired t-tests were also used to compare specific SR points in addition to GEE.

\section{Results}

\section{Analysis of baseline values}

All PSG parameters and PVT scores are shown in Table 1. Baseline values of all parameters did not differ significantly from each other at the start of each SR period, as assessed by paired t-tests. The time courses of changes in PSG parameters over the two 7-day SR trials are shown in Figure 1.

Baseline values of TST prior to SR periods were approximately $400 \mathrm{~min}$ (Table 1). These times were reduced to slightly over $180 \mathrm{~min}$ during SR, and recovered to $482-456 \mathrm{~min}$ after the SR period. Changes in TST did not differ significantly between the two SR protocols, as assessed with GEE analysis $(\mathrm{P}=0.875)$.

\section{Changes of sleep architecture during ESR}

$\mathrm{S} 1 \%$ values remained low and did not change significantly during the four SR nights and two recovery nights (RN1 and RN2). S2\% values decreased notably over the four SR nights, with significant declines from baseline evident in SR2 through SR4 $(\mathrm{P}<0.05)$, before returning to baseline during the two RNs. Levels of S2\% showed a significant negative correlation with the duration of $\mathrm{SR}(\mathrm{r}=-0.941, \mathrm{P}=0.017)$.

SWS\% increased gradually from the first to the fourth SR night. Levels of S4\% increased significantly to similar extents during SR nights, while S3\% only increased significantly on SR4 $(\mathrm{P}=0.004)$. The net increase in SWS\% by SR4 was $132.2 \%$, of which $81.8 \%$ came from the increase in S4\%. All SWS\%, including $\mathrm{S} 4 \%$ and S3\%, recovered to baseline levels in the first and second recovery nights. The time of SWS changed very little in the four SR nights from the SWS time of the normal night sleep, but it was significantly prolonged in the first recovery night $(\mathrm{P}=0.006)$. Significant positive correlations were obtained between SWS\% and days of SR $(r=0.920, p<0.027)$, and between $S 4 \%$ and days of $\mathrm{SR}(\mathrm{r}=0.941, \mathrm{p}=0.017)$.

Values of REM $\%$ decreased significantly in SR1 and SR4 $(\mathrm{P}<0.05)$, but rebounded in the two RNs, especially in the first recovery night $(\mathrm{P}=0.017)$, to levels significantly higher than baseline. The correlation between REM\% and SR duration was not significant.

The L-REM decreased gradually in the four SR nights, but did not differ significantly from baseline. However, it decreased significantly in the first RN $(\mathrm{P}=0.005)$. The correlation between L-REM and the SR duration was significant $(r=-0.893, \mathrm{P}=0.041)$. There was only one sleep onset REM period (SOREMP) in SR4 during the ESR protocol.

\section{Changes of sleep architecture during LSR}

$\mathrm{S} 1 \%$ decreased significantly only in the first RN $(\mathrm{P}=0.048) . \mathrm{S} 2 \%$ decreased markedly during the four $\mathrm{SR}$ nights $(\mathrm{P}<0.01)$, recovered to baseline levels in the two RNs, and showed a significant negative correlation with days of $\mathrm{SR}(\mathrm{r}=-0.956, \mathrm{P}=0.011)$.

SWS\% increased significantly from SR1 to SR4 $(\mathrm{P}<0.01)$. S4\% showed similar levels of increase in all SR nights $(\mathrm{P}<0.01)$, while $\mathrm{S} 3 \%$ increased significantly only in SR4 $(\mathrm{P}=0.037)$. The net increase in $\mathrm{SWS} \%$ over the 4 days of SR was $128.0 \%$, of which $74.1 \%$ was attributable to the increase in S4\%. The total SWS time decreased remarkably only at $\mathrm{SR} 1(\mathrm{P}=0.016)$. It remained at baseline level in the next three SR nights and was significantly prolonged in the recovery nights $(\mathrm{P}<0.05)$. SWS\% and S4\% recovered to baseline levels in the first RN. The correlation between SWS\% and duration of SR 


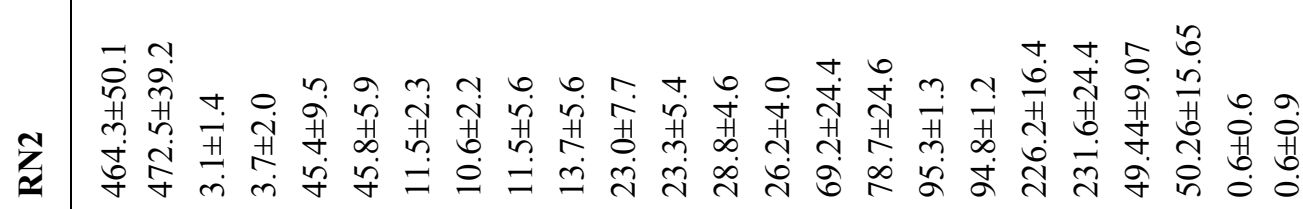

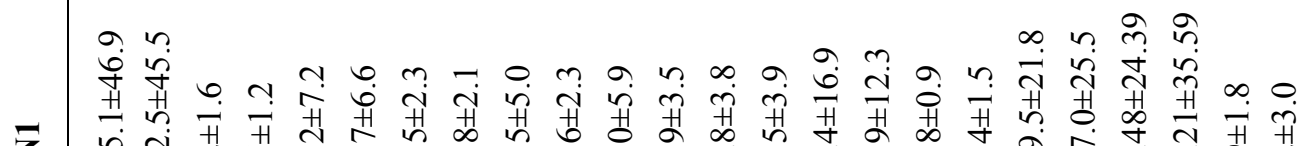

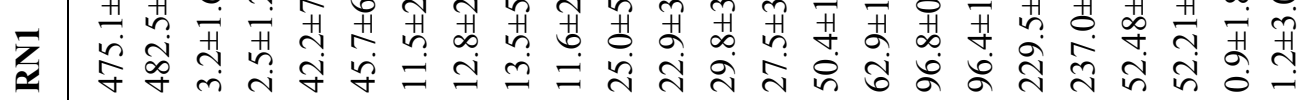

กี

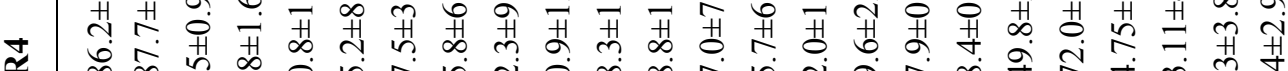

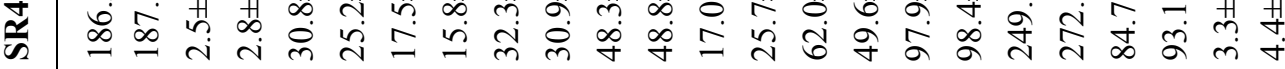

$\approx$

ㅂ.

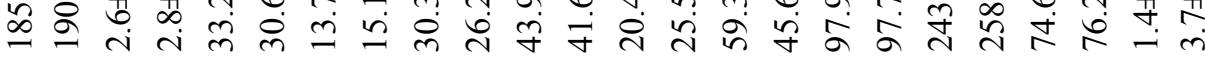

そֶ

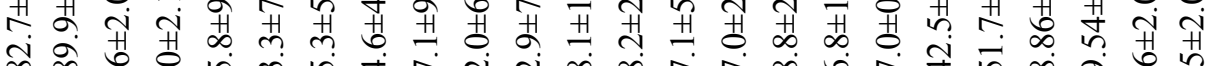

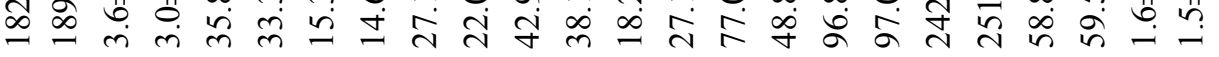

$\ddot{n}+\infty$

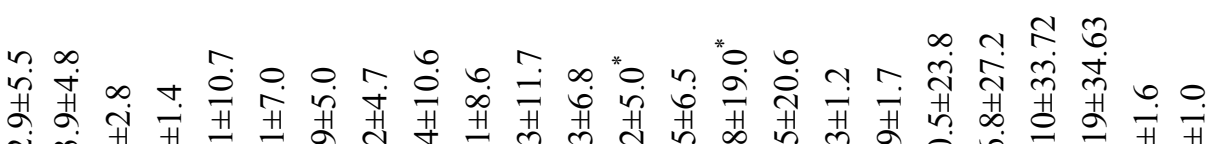

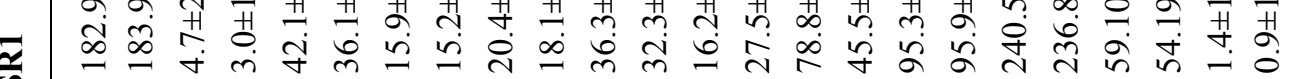

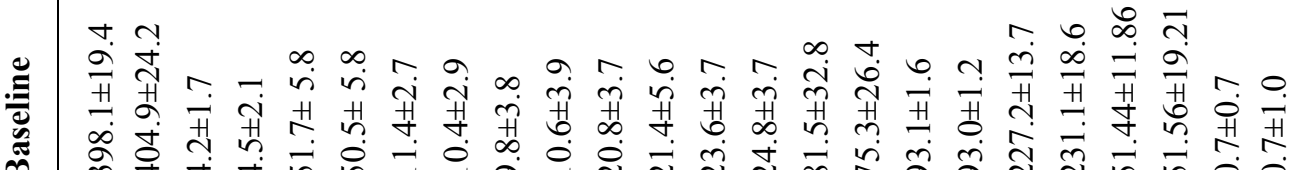

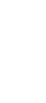

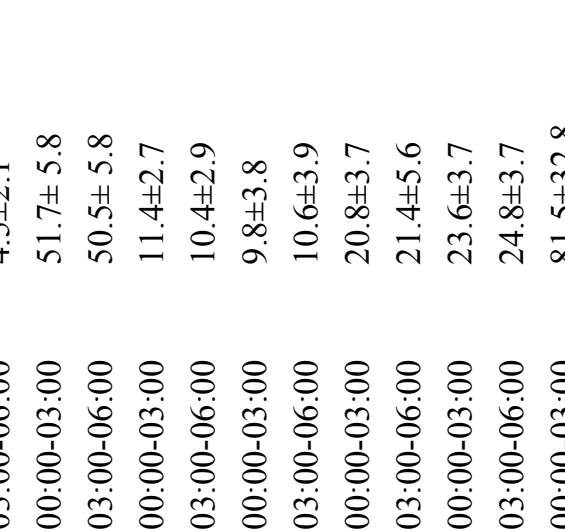

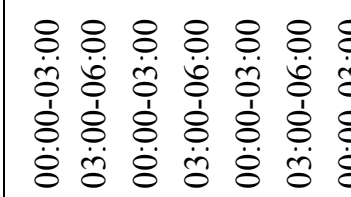

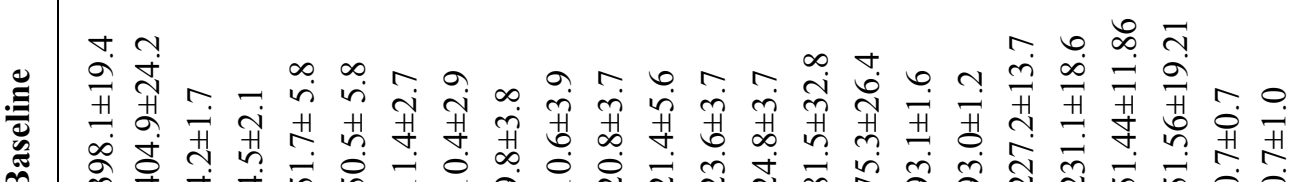

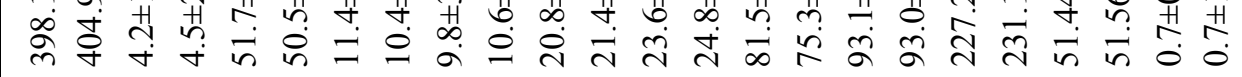

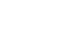

을 믐 亚

言语

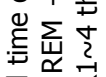

ष्山े

高

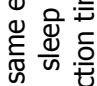

可造

年1 1

o

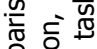

要. 을 
was significant $(\mathrm{r}=0.983, \mathrm{P}=0.003)$, as was the correlation between $\mathrm{S} 4 \%$ and duration of SR $(\mathrm{r}=0.998$, $\mathrm{P}<0.001)$.

REM\% changed slightly during the four SR nights and the two RNs ( $P>0.05)$. The L-REM decreased significantly over the four SR nights $(\mathrm{P}<0.01)$, and recovered to baseline levels in the two RNs. Sleep onset REM periods (SOREMPs) occurred 11 times in the laternight SR trial (6 times on SR1; 3 times on SR2; 1 time on SR3; and once on SR4). Both REM\% and L-REM showed no significant correlation with SR duration.

\section{Statistical comparison between the two SR regimens}

Inter-group analysis by GEE showed only a few significant differences between the two SR regimens, which included REM\% $(\mathrm{z}=3.937, \mathrm{P}<0.001)$ and $\mathrm{L}-\mathrm{REM}$ $(\mathrm{z}=-10.132, \mathrm{P}=0.001)$. Paired t-tests showed significant differences in $\mathrm{REM} \%$ between the two SR regimens for every $S R$ night (SR1: $\mathrm{t}=-6.906, \mathrm{P}<0.001 ; \mathrm{SR} 2$ : $\mathrm{t}=-4.380, \mathrm{P}=0.002 ;$ SR3: $\mathrm{t}=-3.058, \mathrm{P}=0.014 ;$ SR4: $\mathrm{t}=-2.377, \mathrm{P}=0.041$ ), but no significant differences for either of the RNs. Paired t-tests showed significant differences in L-REM between the two SR trials (SR1: $\mathrm{t}=4.699, \quad \mathrm{P}=0.001 ; \quad \mathrm{SR} 2: \mathrm{t}=1.820, \quad \mathrm{P}=0.102 ; \quad \mathrm{SR} 3$ : $\mathrm{t}=2.237, \quad \mathrm{P}=0.052 ; \quad \mathrm{SR} 4: \mathrm{t}=1.067, \quad \mathrm{P}=0.314) . \quad$ The SOREMP ratio between the two SR trials was 11:1. Consistent with the Dunnett t-test analyses, intra-group analysis by GEE showed significant changes from baseline in S1\%, S2\%, and SWS\% in different SR nights within each SR protocol.

\section{Changes of PVT performance during the two SR periods}

During the ESR period, differences in PVT-RTs between baseline level and SR1-4 and RN1-2 did not differ significantly (Dunnett t-test). PVT-lapses only increased significantly from baseline on SR4 $(\mathrm{t}=2.900$, $\mathrm{P}=0.011)$. The number of days of $\mathrm{SR}$ correlated significantly with PVT-RTs $(\mathrm{r}=0.883, \mathrm{P}=0.047)$, but not with lapses $(r=0.796, \mathrm{P}=0.107)$.

During the LSR period, PVT-RTs increased significantly on SR4 $(\mathrm{t}=49.858, \mathrm{P}=0.005)$. PVT-lapses were significantly higher on SR4 $(\mathrm{t}=4.700, \mathrm{P}=0.019)$. Days of SR correlated significantly with PVT-RTs $(\mathrm{r}=0.980, \quad \mathrm{P}=0.003)$ and with lapses $(\mathrm{r}=0.937$, $\mathrm{P}=0.021)$.

GEE analyses did not show significant intergroup differences in PVT-RTs $(Z=2.404, \mathrm{P}=0.121)$ or lapses $(Z=-2.311, P=0.128)$ between the two $S R$ trials. Paired $t$-tests showed longer PVT-RTs $(t=-3.750$,
$\mathrm{P}=0.049$ ) in SR4 in the LSR protocol, but no significant difference between lapses $(\mathrm{t}=-3.188, \mathrm{P}=0.062)$ in SR4. The standard deviations of PVT reaction times were significantly larger on SR4 during LSR than during ESR $(\mathrm{t}=-4.573, \mathrm{P}=0.041)$. Both PVT measures returned to baseline levels after RN1, in both SR regimens.

\section{Discussion}

This study showed several consequences of sleep restriction in healthy adult males. Sleep restriction produced significant increases in SWS in both restriction protocols, significant decreases in REM sleep in the ESR protocol, and longer PVT RTs on the LSR protocol on SR4. All these impairments were reversible. The major findings are summarized in more detail, as follows:

First, better sleep efficiency, as shown by significantly increased percentages of SWS and associated decreases in lighter non-REM sleep (i.e. S1 and S2), was observed in both SR regimens. Consequently, absolute levels of SWS were largely preserved. Moreover, SWS \% was positively correlated with the number of SR nights, while S2\% was negatively correlated. During both sleep regimens, increases in $\mathrm{S} 4 \%$ were the main factor underlying increases in SWS\%.

A second important consequence of the SR regimens involves the significant differential effects on REM sleep. The reduction of REM sleep in earlier-night SR was more notable than it was in later-night SR. During the earlier-night SR period, REM\% dropped significantly, but then rebounded to exceed baseline levels in the first RN. By contrast, REM\% was maintained near baseline levels in the later-night SR and recovery periods. Similarly, L-REM changed as a function of the $\mathrm{SR}$ regimen. It decreased moderately during the earlier-night regimen, with one SOREMP, but showed a greater decrease during the later-night SR, with 11 SOREMPs.

Sleep architecture is composed of the succession of cycles, their component stages, and the duration of each stage and cycle. It is generated by an interplay of multiple external and internal oscillators, which is hypothesized to include circadian modulation, homeostatic systems and ultradian rhythms (Benington and Heller 1999, Borbély and Achermann 1999, Merica and Fortune 2004, Sinton and McCarley 2004). These later rhythms are shorter than circadian rhythms, and are hypothesized to influence 90-min cycles of NREM and REM sleep during the night (Capitani et al. 2005, 
Kräuchi et al. 2006). Nocturnal sleep is artificially divided into two parts: earlier-night sleep that is rich in SWS, and later-night sleep that is rich in REM sleep (Brunner et al. 1990). This phenomenon is consistent with our division of normal sleep periods for the baseline preceding SR into two components. Data from our study also confirmed that, when sleep time had to be shortened, the remaining percentage of each sleep stage reflected the need for those stages of sleep. The retention of SWS during sleep restriction supports the view that SWS appears to be the 'core sleep' (Tilley and Wilkinson 1984). REM sleep is next in importance in sleep homeostasis, while lighter NREM sleep (S1 and S2) may be sacrificed when sleep time is restricted. Differences between two SR protocols on changes of REM\% and L-REM are consistent with this view. It is likely that both homeostatic and circadian influences contributed to these findings (Borbély and Achermann 1999). However, the persistent differences in REM $\%$ between the ESR and LSR conditions from SR1 to SR4 may also reflect ultradian influences. Moreover, as our earlier study with the same subjects showed, hormonal differences involving cortisol secretion (and probably other hormones of arousal) likely had direct or indirect modulating effects as well (Wu et al. 2008).

The third major effect of sleep restriction involves its negative effects on daytime vigilance. Both SR regimens were associated with increasingly impaired daytime vigilance, as measured by increased reaction times and performance lapses on a straightforward vigilance task. Cognitive functions are closely related to the amount and quality of sleep. Some researchers have proposed that mild sleep restriction does not significantly affect vigilance (Binks et al. 1999). However, other researchers proposed that even mild sleep deprivation impairs cognition significantly (Banks and Dinges 2007, Belenky et al. 2003, Elmenhorst et al. 2008, Van Dongen et al. 2003). Our data supported the view that SR results in increasingly impaired neurobehavioral performance. However, the reaction times of PVT performance were affected more seriously during LSR than during ESR. Because of more microsleep during LSR, the deviation of PVT-RTs was significantly larger during LSR than it was during ESR. Earlier-night sleep may thus play a more important role in protecting cognition function, although this hypothesis was not assessed directly in this study. Moreover, the roughly equal levels of TST and SWS time in both SR regimens, along with the retention of absolute levels of SWS, emphasize the importance of TST, rather than just some of its components (i.e. SWS and REM), in maintaining normal cognitive performance (see also Tilley and Wilkinson 1984).

Finally, this study showed that values of sleep architecture and PVT performance recovered to baseline levels after one night of recovery sleep. It also shows significant REM sleep debt in the first recovery night. Consistent with other studies employing comparable designs (Elmenhorst et al. 2008), the current findings emphasize the effectiveness of recovery night sleep in reversing both sleep and cognitive deficits after one recovery night. This recovery was evident in both SR conditions, and also applied to SR-induced changes in cortisol levels, as we reported previously (Wu et al. 2008). These findings have several implications. One is to emphasize the importance of sleep in maintaining normal function across a range of physiological and cognitive dimensions of function. Similarly, the ability of recovery sleep to restore function quickly and effectively suggests that effects of sleep deprivation are due to the loss of sleep and are not only secondary consequences of disturbed function in different domains (e.g. hormonal function). It will be important to determine the effectiveness and parameters of recovery sleep in more chronic sleep restriction paradigms. A second implication is that the effects of recovery sleep are not only palliative but are also preventive, which highlights the growing importance of proper sleep hygiene in modern societies.

The design used in this study has some limitations, which include, among others, a focus on males only, and a restricted age range. Moreover, the number of subjects was relatively small. Because we intended to establish main effects associated with sleep and other physiological measures (Wu et al. 2008) associated with the SR paradigms with this small sample, we did not also counterbalance the order of the two SR protocols to assess potential effects of receiving ESR prior to LSR, or perform corrections for multiple statistical tests. This limits the extent to which the current findings can be generalized, and underscores the need for additional larger studies with adequate statistical power to assess how order effects and other variables modulate effects of SR protocols.

Overall, our data show the evidence of both immediate (i.e. evident in the first night) and progressive changes in nocturnal sleep in two different SR protocols. Although some of these effects were modest (e.g. PVT lapses did not become significant until SR4), they are clinically significant in the context of societies that are 
increasingly afflicted by chronic partial sleep deprivation. In this view, the significant lapses in attention demonstrated after moderate sleep reductions by SR4 show that cognitive decrements occur relatively soon in chronic sleep-deprived populations, even in healthy, young adult individuals. However, these data are equally important in demonstrating the restorative properties of recovery sleep.

The current study shows that the timing of SR is significant, as earlier-night sleep deprivation impaired daytime vigilance more strongly than later-night deprivation. It will be important to establish these effects on additional, more complex cognitive functions (such as declarative memory and executive functions such as organization, problem-solving, resistance to distraction and fluency, among others), and on daytime sleepiness and physiological measures of arousal. As noted above, the current findings will also need to be validated in larger samples, and in longer SR paradigms. Eventually, naturalistic designs in vulnerable populations (e.g. shift workers) may demonstrate the extent to which different
SR periods affect cognition and other dimensions of daily function. Despite these caveats, these findings add to and underscore mounting evidence that partial sleep deprivation results in progressive, negative effects in neurobehavioral performance. These effects occur in healthy young adults, and reflect a growing toll of sleep deprivation in modern societies.

\section{Conflict of Interest}

There is no conflict of interest.

\section{Acknowledgements}

We thank Wei Dong for his help in data analysis and statistics, and the subjects for their participation. This work was supported by grants from the specific health issues projects of the Ministry of Health, General Logistics Department of the Chinese People's Liberation Army (07BJZ06), the Shanghai Science and Technology Development Foundation (08411950700) and Medicine Importance Foundation (09DZ1950400) from Science and Technology Commission of Shanghai Municipality.

\section{References}

ANDERSON C, HORNE JA: Sleepiness enhances distraction during a monotonous task. Sleep 29: 573-576, 2006.

BANKS S, DINGES DF: Behavioral and physiological consequences of sleep restriction. J Clin Sleep Med 3: 519-528, 2007.

BELENKY G, WESENSTEN NJ, THORNE DR, THOMAS ML, SING HC, REDMOND DP, RUSSO MB, BALKIN TJ: Patterns of performance degradation and restoration during sleep restriction and subsequent recovery: a sleep dose-response study. $J$ Sleep Res 12: 11-12, 2003.

BENINGTON JH, HELLER HC: Implications of sleep deprivation experiments for our understanding of sleep homeostasis. Sleep 22: 1033-1037, 1999.

BINKS PG, WATERS WF, HURRY M: Short-term total sleep deprivations does not selectively impair higher cortical functioning. Sleep 22: 328-334, 1999.

BONNET MH, ARAND DL: We are chronically sleep deprived. Sleep 18: 908-911, 1995.

BORBÉLY AA, ACHERMANN P: Sleep homeostasis and models of sleep regulation. $J$ Biol Rhythms 14: 557-568, 1999.

BRUNNER DP, DIJK DJ, TOBLER I, BORBÉLY AA: Effect of partial sleep deprivation on sleep stages and EEG power spectra: evidence for non-REM and REM sleep homeostasis. Electroencephalogr Clin Neurophysiol 75: 492-499, 1990.

CAPITANI P, CERRI M, AMICI R, BARACCHI F, JONES CA, LUPPI M, PEREZ E, PARMEGGIANI PL, ZAMBONI G: Changes in EEG activity and hypothalamic temperature as indices for non-REM sleep to REM sleep transitions. Neurosci Lett 383: 182-187, 2005.

DIJK DJ, DUFFY JF, CZEISLER CA: Circadian and sleep/wake dependent aspects of subjective alertness and cognitive performance. J Sleep Res 1: 112-117, 1992.

DINGES DF, POWELL JW: Microcomputer analyses of performance on a portable, simple visual RT task during sustained operations. Behav Res Meth Instrum Comput 17: 652-655, 1985.

ELMENHORST EM, ELMENHORST D, LUKS N, MAASS H, VEJVODA M, SAMEL A: Partial sleep deprivation: Impact on the architecture and quality of sleep. Sleep Med 9: 840-850, 2008. 
GREENE R, SIEGEL J: Sleep: a functional enigma. Neuromolecular Med 5: 59-68, 2004.

GUILLEMINAULT C, POWELL NB, MARTINEZ S, KUSHIDA C, RAFFRAY T, PALOMBINI L, PHILIP P: Preliminary observations on the effects of sleep time in a sleep restriction paradigm. Sleep Med 4: 177-184, 2003.

HANLEY JA, NEGASSA A, EDWARDES MD, FORRESTER JE: Statistical analysis of correlated data using generalized estimating equations: an orientation. Am. J Epidemiol 157: 364-375, 2003.

KRÄUCHI K, CAJOCHEN C, PACHE M, FLAMMER J, WIRZ-JUSTICE A: Thermoregulatory effects of melatonin in relation to sleepiness. Chronobiol Int 23: 475-484, 2006.

MERICA H, FORTUNE RD: State transitions between wake and sleep, and within the ultradian cycle, with focus on the link to neuronal activity. Sleep Med Rev 8: 473-485, 2004.

NATIONAL SLEEP FOUNDATION. 2003 Sleep in America Poll. Washington DC: National Sleep Foundation; 2003. http://www.sleepfoundation.org/2003poll.cfm

PHILIP P, TAILLARD J, SAGASPE P, VALTAT C, SANCHEZ-ORTUNO M, MOORE N, CHARLES A, BIOULAC B: Age, performance and sleep deprivation. J Sleep Res 13: 105-110, 2004.

RECHTSCHAFFEN A, KALES A (eds): A Manual of Standardized Terminology, Techniques and Scoring System for Sleep Stages of Human Subjects. US Department of Health, Education and Welfare, Public Health Service, Bethesda, MD, 1968.

SINTON CM, MCCARLEY RW: Neurophysiological mechanisms of sleep and wakefulness: a question of balance. Semin Neurol 24: 211-223. 2004.

TILLEY AJ, WILKINSON RT: The effects of a restricted sleep regimen on the composition of sleep and on performance. Psychophysiology 21: 406-412, 1984.

VAN DONGEN HP, MAISLIN G, MULLINGTON JM, DINGES DF: The cumulative cost of additional wakefulness: dose-response effects on neurobehavioral functions and sleep physiology from chronic sleep restriction and total sleep deprivation. Sleep 26: 117-126, 2003.

WEBB WB, AGNEW HW: The effects of a chronic limitation of sleep length. Psychophysiology 11: 265-274, 1974.

WU H, ZHAO Z, STONE WS, HUANG L, ZHUANG J, HE B, ZHANG P, LI Y: Effects of sleep restriction periods on serum cortisol levels in healthy men. Brain Res Bull 77: 241-245, 2008. 